\title{
Cóndilo Mandibular Bífido: Un Desafío Diagnóstico
}

\author{
Bífid Mandibular Condyle: a Diagnostic Challenge \\ Frederico Sampaio Neves; Laura Ricardina Ramirez-Sotelo; Deborah Queiroz de Freitas; \\ Francisco Haiter-Neto \& Frab Norberto Bóscolo
}

NEVES, F. S.; RAMIREZ-SOTELO, L. R.; FREITAS, D. Q.; HAITER-NETO, F. \& BÓSCOLO, F. N. Cóndilo Mandibular Bífido: Un desafío diagnóstico. Int. J. Odontostomat., 6(3):327-330, 2012.

RESUMEN: El cóndilo mandibular bífido es una condición rara, observada frecuentemente como hallazgo radiográfico, caracterizada por la división de la cabeza de la mandíbula. Su prevalencia varía entre 0,0018\% a 0,3\%, donde múltiples causas han sido sugeridas en la literatura para justificar su desarrollo, sin embargo no hay concordancia entre los estudios acerca del factor causal principal. El objetivo de este artículo es relatar un caso de cóndilo mandibular bífido observado en una mujer de 21 años de edad, el cual fue revelado por una radiografía panorámica donde se observó hipoplasia del cóndilo mandibular izquierdo, con presencia de una aparente calcificación en la región de la articulación temporomandibular izquierda; otros exámenes convencionales fueron realizados (radiografía postero-anterior, con apoyo frente-nasion en boca abierta, transcraniana y panorámica), sin embargo, apenas en la tomografía computarizada de haz cónico, fue posible obtener el diagnóstico de cóndilo mandibular bífido.

PALABRAS CLAVE: Cóndilo mandibular bífido, articulación temporomandibular, tomografía computarizada.

\section{INTRODUCCIÓN}

El cóndilo mandibular bífido (CMB) es una condición rara, caracterizada por la división de la cabeza de la mandíbula. Según la literatura consultada, la prevalencia encontrada varía de $0,018 \%$ a 0,3\% (Menezes et al., 2008; Miloglu et al., 2010).

Las dos superficies articulares del cóndilo bífido pueden ser divididas por un surco orientado mediolateralmente o anteroposteriormente. La división del cóndilo puede variar desde un surco superficial hasta la formación de dos cabezas de cóndilo distintas (Miloglu et al.).

Apesar de que algunos casos pueden estar asociados a la anquilosis de la articulación temporomandibular (ATM) o a la presencia de sonidos articulares (Stefanou et al., 1998; Ramos et al., 2006), la mayoría de los pacientes es asintomático, y los casos de CMB son hallazgos incidentales en exámenes radiográficos convencionales, tomografía computarizada o resonancia magnética de la cabeza y cuello (McCormick et al., 1989).
El objetivo de este artículo es relatar los aspectos clínicos e imaginológicos de un caso de cóndilo mandibular bífido.

\section{RELATO DE CASO}

Paciente de sexo femenino, 21 años, acudió al Servicio de radiología oral de la Universidad Estadual de Campinas - Brasil, para realización de radiografías dentarias con finalidad de extracción de terceros molares. En la radiografía panorámica, se observó hipoplasia del cóndilo mandibular izquierdo, con presencia de una aparente calcificación en la región de la ATM del lado izquierdo (Fig. 1).

La paciente relato historia de trauma en la infancia, que produjo fractura condilar, situación que fue acompañada con exámenes radiográficos, sin la realización de intervención quirúrgica. La paciente no reporto sintomatología dolorosa y al examen físico, no fueron 
observadas limitaciones de apertura bucal o de presencia de sonidos articulares; sin embargo, había un discreto desvío de la línea media hacia el lado izquierdo.

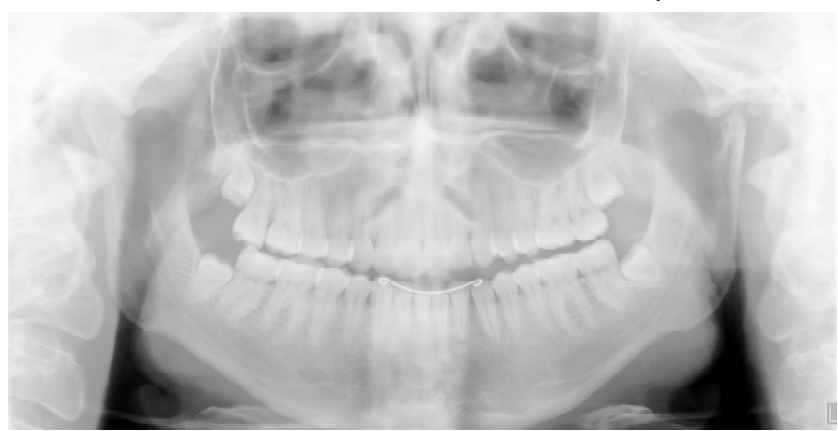

Fig. 1. Radiografía panorámica, evidenciando hipoplasia del cóndilo mandibular izquierdo con presencia de una aparente calcificación en la región de la ATM izquierda.

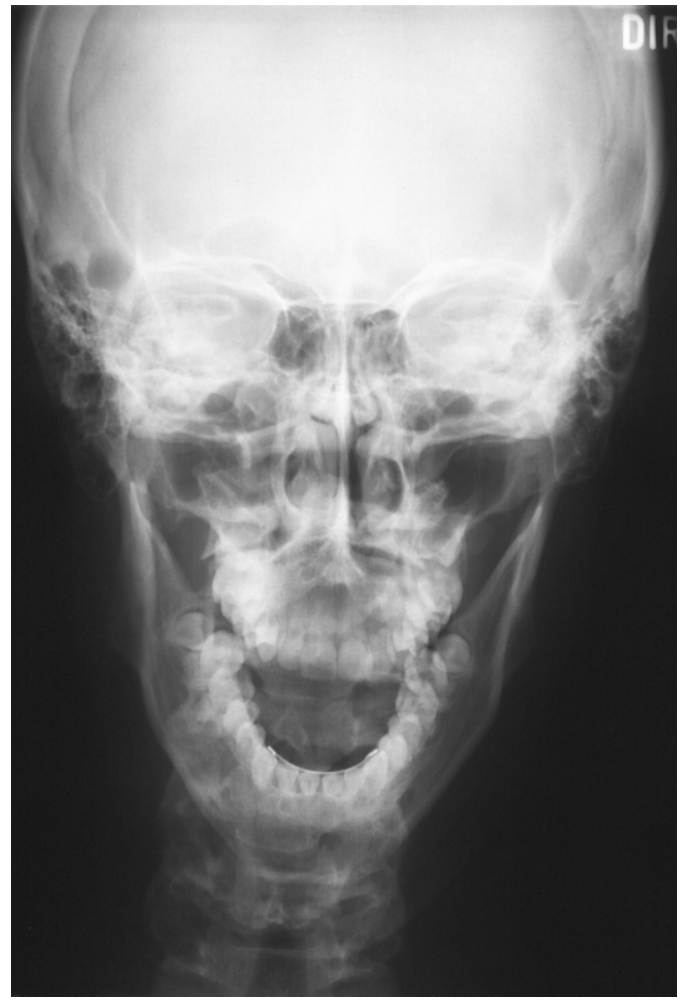

Fig. 2. Radiografía postero-anterior, con apoyo frente-nasion, a boca abierta evidenciando el desvío de la línea mediana para el lado izquierdo, pero sin alteración condilar evidente.

En la radiografía postero-anterior, con apoyo frente-nasion, en boca abierta (Fig. 2), también se observó el desvío de la línea mediana para la izquierda; entretanto no fue encontrada ninguna alteración condilar. En la radiografía transcraniana (Fig. 3), de igual forma no fueron observadas alteraciones significativas.
Como en la radiografía panorámica fue visualizada una aparente calcificación en la ATM izquierda y la misma condición no fue encontrada en las demás imágenes convencionales realizadas, no fue posible la elaboración de un diagnóstico conclusivo, la paciente fue sometida a un examen por tomografía compu-tarizada de haz cónico (i-CAT, Imaging Sciences International, Inc, Hatfield, PA, EUA), operando a $120 \mathrm{kVp}, 8 \mathrm{~mA}$ y tamaño de voxel $0,25 \mathrm{~mm}$. En los cortes coronales, fue observada la imagen del cóndilo bífido, del lado izquierdo, con orientación media-lateral; la porción lateral del cóndilo presentó un facetamiento y mayor dimensión; sin embargo, se observó la presencia de apenas una fosa articular (Fig. 4). Como la paciente no presentaba signos/síntomas, ningún tratamiento fue instituido.

\section{DISCUSIÓN}

Varios orígenes han sido sugeridos en la literatura para el desarrollo del CMB, sin embargo no hay acuerdo entre los estudios acerca del factor causante principal. El origen genético de tal anormalidad ósea ha sido investigada, pero pequeños traumas o factores de desarrollo intrauterinos o durante la infancia han sido demostrados como los más significativos. Antoniades et al. (1993), sugirieron que el desarrollo del CMB es causado debido a una capacidad de remodelación óssea insuficiente. Quayle \& Adams (1986), indicaron que desordenes endocrinas, deficiencia nutricional, infección, trauma, irradiación y factores genéticos pueden ser posibles factores causales. Thomason \& Yusul (1986), relataron dos casos de fractura condilar de origen traumático, por accidente de bicicleta, que acarreó en la formación de un cóndilo bífido. Tal relación es corroborada con los hallazgos de Poswillo (1972), que observó relación entre la formación del cóndilo bífido e historia de trauma. En el caso presentado en este articulo, la paciente relató trauma en la infancia, siendo ese el probable factor causal para la formación del CMB.

La prevalencia del CMB es extremadamente baja Miloglu et al., evaluaron 10200 radiografías panorámicas de población Turca y detectaron sólo 32 casos $(0,3 \%)$ de CMB, siendo 24 casos $(75 \%)$ unilaterales y 8 casos $(25 \%)$ bilaterales. Menezes et al. examinaron 50800 radiografías panorámicas de la populación brasileira, observaron sólo 9 casos $(0,018 \%)$ de CMB, 7 unilaterales (78\%) y 2 bilaterales (22\%). En ambos estudios, todos los pacientes negaron historia previa de trauma. La ocurrencia de cóndilo mandibular trífido es aun más rara, siendo apenas dos casos relatados en la literatura (Artvinli \& Kansu, 2003; Cagirankaya \& Hatipoglu, 2005). 

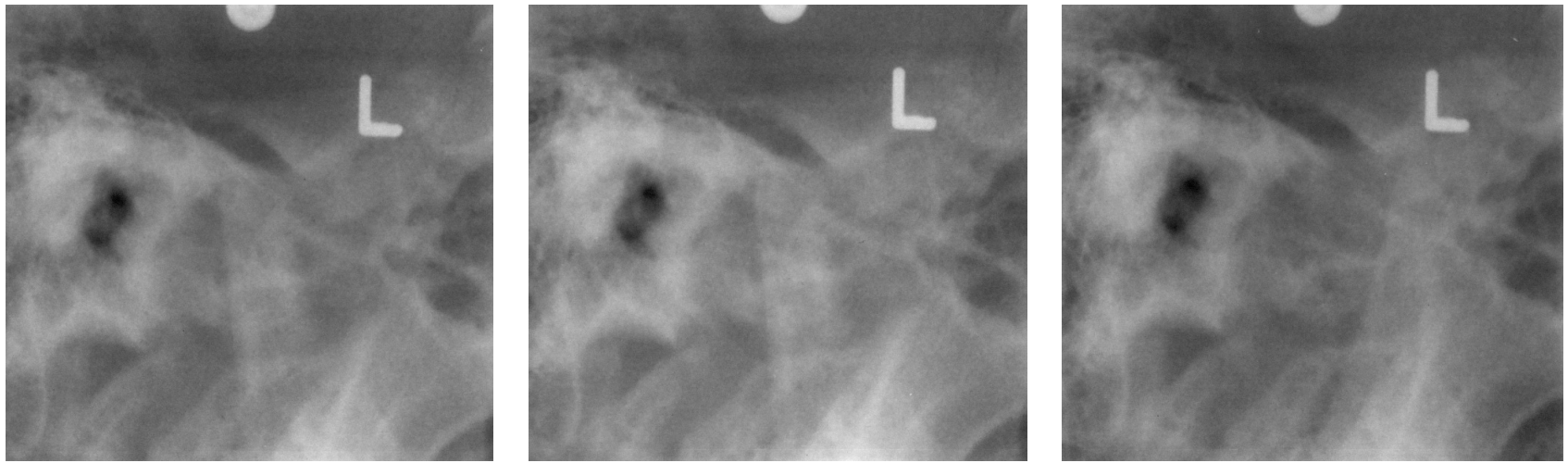

Fig. 3. Radiografía transcraniana de la ATM izquierda, en la cual no fueron observadas alteraciones significativas.

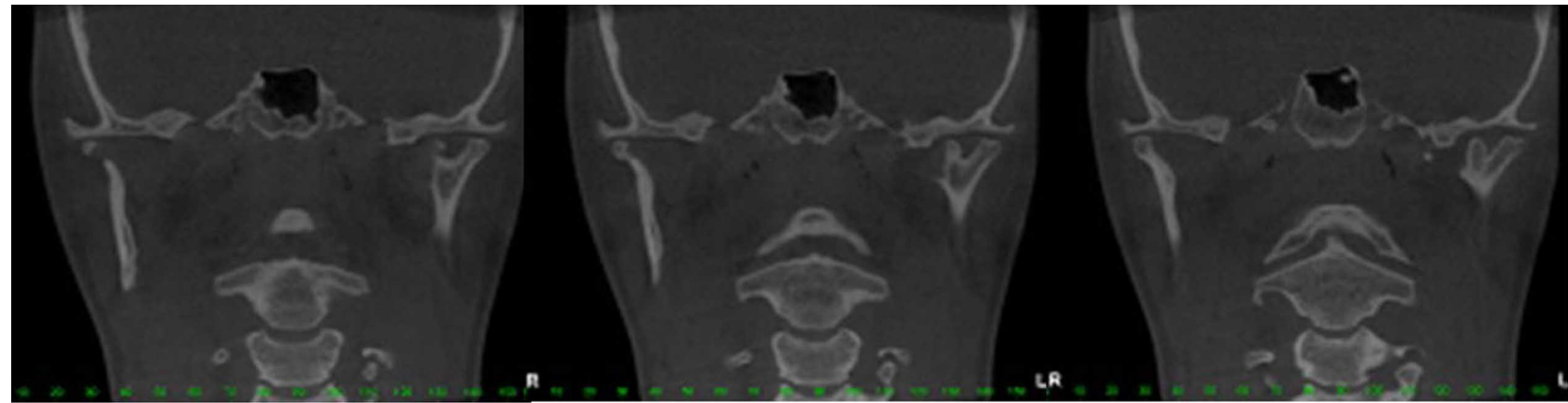

Fig. 4. Cortes coronales que evidencian la imagen de cóndilo bífido.

Los síntomas asociados a la presencia del CMB son variables, sin embargo, la gran mayoría de los casos se muestra asintomático (McCormick et al.; Fernández et al., 2009) cuando presentes, los más comunes son sonidos articulares (Sales et al., 2007). Pero raramente, dolor articular (McCormick et al.; Rehman et al., 2009) y anquilosis (Stefanou et al.; Ramos et al.; Balaji, 2009) son relatados. En el presente caso, ninguna sintomatología estaba asociada al CMB.

La morfología del CMB puede variar de un surco superficial a la presencia de dos cabezas condilares distintas. La orientación de las dos cabezas condilares puede variar entre dos padrones: medio-lateral y antero-posterior. La existencia de esos dos patrones de CMB pode estar relacionada al factor causal. Segun Szentpetery et al. (1990), el CMB en el sentido antero-posterior resultaría de trauma en la infancia, mientras que en el sentido medio-lateral se desarrolla debido a la permanencia de un tabique o septo fibroso en el cartílago condilar. Sin embargo, Cowan \& Fergusson (1997) defendieron que el factor causante no influencia el sentido del cóndilo bífido, lo que se corrobora con el caso relatado, visto que la paciente relató historia de trauma en la infancia, e el CMB se presentó en sentido medio-lateral.

De acuerdo con Dennison et al. (2008), el térmi- no "cóndilo bífido" es frequentemente utilizado en la descripción de edentaciones o surcos profundos en el cóndilo mandibular. Pero tal terminología debe ser utilizada solo cuando ocurre la presencia de múltiples cóndilos en los planos sagital, coronal y axial. Por tanto, a partir de las imágenes tomográficas observadas en el presente caso, se le puede considerar como CMB verdadero.

El tratamiento apropiado para los casos de CMB depende exclusivamente de los síntomas presentados por el paciente. Para los casos sintomáticos, el tratamiento es el mismo utilizado en pacientes con desordenes temporomandibulares, en que se opta por la administración de analgésicos, antiinflamatorios, relajantes musculares y fisioterapia. Para los casos de anquilosis de la articulación, el tratamiento quirúrgico es el de primera elección (Antoniades et al.; Corchero-Martín et al., 2005). Debido a la ausencia de sintomatología por parte de la paciente, ningún tratamiento fue instituido para el caso.

En el presente caso, las radiografías convencionales no se mostraron eficaces en el diagnóstico de CMB; además de eso, si la radiografía panorámica fuese evaluada individualmente, nos conduziria a un diagnóstico erróneo para el caso. Las imágenes tomográficas fueron determinantes em el diagnóstico final de esta condición. 
NEVES, F. S.; RAMIREZ-SOTELO, L. R.; FREITAS, D. Q.; HAITER-NETO, F. \& BÓSCOLO, F. N. Bífid Mandibular Condyle: a diagnostic challenge. Int. J. Odontostomat., 6(3):327-330, 2012.

ABSTRACT: The mandibular bifid condyle is a rare condition, frequently observed in routine X-rays, characterized by division of the mandible head. According to the literature, the prevalence varies from $0.018 \%$ to $0.3 \%$. Several causes have been suggested to justify the development of bifid mandibular condyle, however there is no agreement between studies about the main causative factor. The aim of this paper is to report a case of mandibular bifid condyle in a 21-years-old girl. The panoramic radiograph showed hypoplasia of the left mandibular condyle, with an apparent presence of calcification in the left temporomandibular joint. Other conventional extraoral radiographs were performed (postero-anterior with fronto-nasal support, open mouth; panoramic and trascranial radiographs), however, only in the cone beam computed tomography ir was possible to obtain the diagnosis of mandibular bifid condyle.

KEY WORDS: mandibular bifid condyle, temporomandibular joint, computed tomography.

\section{REFERENCIAS BIBLIOGRÁFICAS}

Antoniades, K.; Karakasis, D. \& Elephtheriades, J. Bifid mandibular condyle resulting from a sagittal fracture of the condylar head. Br. J. Oral Maxillofac. Surg.,31(2):124-6, 1993.

Artvinli, L. B. \& Kansu, O. Trifid mandibular condyle: A case report. Oral Surg. Oral Med. Oral Pathol. Oral Radiol. Endod., 95(2):251-4, 2003.

Balaji, S. M. Bifid mandibular condyle with tempromandibular joint ankylosis - a pooled data analysis. Dent. Traumatol., 26(4):3327, 2010.

Cagirankaya, L. B. \& Hatipoglu, M. G. Trifid mandibular condyle: a case report. Cranio, 23(4):297-9,2005.

Corchero-Martín, G.; Gonzalez-Terán, T.; García-Reija, M. F.; Sánchez-Santolino, S. \& Saiz-Bustillo, R. Bifid condyle: case report. Med. Oral Patol. Oral Cir. Bucal, 10(3):277-9, 2005.

Cowan, D. F. \& Ferguson, M. M. Bifid mandibular condyle. Dentomaxillofac. Radiol., 26(1):70-3, 1997.

Dennison, J.; Mahoney, P.; Herbison, P. \& Dias, G. The false and the true bifid condyles. Homo, 59(2):149-59, 2008.

Fernández,R. F.; Flores, H. F.; Mella, H. S. \& Lillo, T. F. Bifid condilar process:case report. Int. J. Morphol., 27(2):539-41, 2009.

Loh, F. C. \& Yeo, J. F. Bifid mandibular condyle. Oral Surg. Oral Med. Oral Pathol., 69(1):24-7, 1990.

McCormick, S. U.; McCormick, S. A.; Graves, R. W. \& Pifer, R. G. Bilateral bifid mandibular condyles. Report of three cases. Oral Surg. Oral Med. Oral Pathol., 68(5):555-7, 1989.

Menezes, A. V.; de Moraes Ramos, F. M.; de Vasconcelos-Filho, J. O.; Kurita, L. M.; de Almeida, S. M. \& Haiter-Neto, F. The prevalence of bifid mandibular condyle detected in a Brazilian population. Dentomaxillofac. Radiol., 37(4):220-3, 2008.

Miloglu, O.; Yalcin, E.; Buyukkurt, M.; Yilmaz, A. \& Harorli, A. The frequency of bifid mandibular condyle in a Turkish patient population. Dentomaxillofac. Radiol., 39(1):42-6, 2010.
Poswillo, D. E. The late effects of mandibular condylectomy. Oral Surg. Oral Med. Oral Pathol., 33(4):500-12, 1972.

Quayle, A. A. \& Adams, J. E. Supplemental mandibular condyle. Br. J. Oral Maxillofac. Surg., 24(5):349-56, 1986.

Ramos, F. M.; Filho, J. O.; Manzi, F. R.; Bóscolo, F. N. \& Almeida, S. M. Bifid mandibular condyle: a case report. J. Oral Sci., 48(1):35-7, 2006.

Rehman, T. A.; Gibikote, S.; Ilango, N.; Thaj, J.; Sarawagi, R. \& Gupta, A. Bifid mandibular condyle with associated temporomandibular joint ankylosis: a computed tomography study of the patterns and morphological variations. Dentomaxillofac. Radiol., 38(4):239-44, 2009.

Sales, M. A.; Oliveira, J. X. \& Cavalcanti, M. G. Computed tomography imaging findings of simultaneous bifid mandibular condyle and temporomandibular joint ankylosis: case report. Braz. Dent. J., 18(1):74-7, 2007.

Stefanou, E. P.; Fanourakis, I. G.; Vlastos, K. \& Katerelou, J. Bilateral bifid mandibular condyles. Report of four cases. Dentomaxillofac. Radiol., 27(3):186-8, 1998.

Szentpétery, A.; Kocsis, G. \& Marcsik, A. The problem of the bifid mandibular condyle. J. Oral Maxillofac. Surg., 48(12):1254-7, 1990.

Thomason, J. M. \& Yusuf, H. Traumatically induced bifid mandibular condyle: a report of two cases. Br. Dent. J., 161(8):291-3, 1986.

Dirección para Correspondencia:

Frederico Sampaio Neves

Departamento de Diagnóstico Oral

Disciplina de Radiologia Odontológica, Avenida Limeira, 901,

Caixa Postal 52

Piracicaba, São Paulo, CEP 13414-903

BRASIL

Telefóno: +55 (19) 21065327

Email: fredsampaio@yahoo.com.br

Recibido : 12-04-2012

Aceptado: 28-08-2012 